\title{
Tratamento cirúrgico dos aneurismas da aorta abdominal: existe diferença dos resultados entre homens e mulheres?
}

\author{
Surgical treatment of abdominal aortic aneurysms: \\ is there difference in the results obtained in men and women?
}

Telmo Pedro Bonamigo', Márcio Luís Lucas', Nilon Erling Jr. ${ }^{3}$

\begin{abstract}
Resumo
O bjetivo: Avaliar os resultados cirúrgicos após o tratamento convencional e eletivo do aneurisma da aorta abdominal, verificando se existe diferença entre homens e mulheres na mortalidade e morbidade cirúrgicas, bem como nos resultados a longo prazo.

Pacientes e métodos: Entre dezembro de 1983 e dezembro de 2003, 675 pacientes foram submetidos ao tratamento cirúrgico do aneurisma da aorta abdominal infra-renal, sendo divididos em dois grupos: homens ( $n=575)$ e mulheres $(n=100)$. O s dados demográficos e aqueles relacionados ao procedimento cirúrgico, bem como os resultados perioperatórios, foram coletados pela revisão dos prontuários. 0 s pacientes com alta hospitalar formaram uma coorte retrospectiva, onde foram avaliadas as causas tardias de óbito e a sobrevida.

Resultados: A média das idades foi similar, sem diferença estatística entre mulheres e homens $(68,9 \pm 9,1$ versus $67,4 \pm 7,1$ anos; $P=0,089)$. A presença de hipertensão arterial foi significativamente superior nas mulheres ( 73 versus $62,4 \% ; P=0,042$ ), e a cardiopatia isquêmica e história de tabagismo foram mais freqüentes nos homens ( $P<0,05)$. A mortalidade operatória total foi de $2,8 \%$, não havendo diferença significativa entre mulheres e homens (4 versus $2,6 \%$, respectivamente; $P=0,43$ ). A morbidade cirúrgica envolveu $14 \%$ dos pacientes femininos e $18,4 \%$ dos masculinos $(P>0,05)$. A taxa de sobrevida global em 1, 3, 5 e 10 anos não diferiu entre os grupos, sendo a sobrevida em 5 anos de $71 \%$ para as mulheres e $72 \%$ para os homens $(P>0,05)$. A principal causa de morte tardia foi de origem cardiovascular, seguida de complicações renais nas mulheres e neoplasia nos homens.

Conclusão: A taxa de mortalidade e morbidade cirúrgicas não aumentou significativamente nas mulheres após a correção el etiva do aneurisma da aorta abdominal infra-renal. Além disso, os resultados a longo prazo são semel hantes entre os dois gêneros.
\end{abstract}

Palavras-chave: Aneurisma da aorta abdominal, cirurgia, mulheres.

\begin{abstract}
0 bjective: To evaluate perioperative results in patients submitted to elective conventional open repair of abdominal aortic aneurysm, comparing the results between women and men in relation to perioperative mortality and morbidity, as well as long term outcomes.

Patients and methods: Between December 1983 and D ecember 2003, 675 patients were submitted to infrarenal abdominal aortic aneurysm repair. We divided these patients into two groups: men ( $n=$ 575 ) and women $(n=100)$. D emographic and operative data, as well as perioperative outcomes were obtained from chart review. $D$ ischarged patients formed a retrospective cohort, in which the late causes of death and survival were evaluated.

Results: The mean age was similar, but no statistical difference was observed between men and women ( $68.9 \pm 9.1$ versus. $67.4 \pm 7.1$ years; $P=0.089)$. T he presence of hypertension was significantly higher in women (73 versus $62.4 \% ; P=0.042$ ), and coronary artery disease and history of smoking were more prevalent in men $(P<0.05)$. The overall mortality rate was $2.8 \%$, without any significant difference between women and men (4 versus $2.6 \%$, respectively; $P=0.43$ ). Perioperative morbidity was similar in both groups ( $14 \%$ for women; $18.4 \%$ for men; $P>0.05)$. The survival rates in $1,3,5$, and 10 years were similar in the groups, with a 5 -year survival of $71 \%$ for women and $72 \%$ for men ( $P>0.05)$. Cardiovascular disease was the main late death cause in both groups, followed by renal complications in women and neoplasia in men.

Conclusion: Perioperative mortality and morbidity rates did not significantly increase in women after conventional infrarenal abdominal aortic aneurysm repair. Furthermore, long term results were similar in both genders.
\end{abstract}

Key words: Abdominal aortic aneurysm, surgery, women.

\footnotetext{
1. Chefe, Serviço de Angiologia e Cirurgia Vascular, Complexo Hospitalar Santa Casa de Porto Alegre, Porto Alegre, RS. Professor adjunto de Cirurgia Vascular, Fundação Faculdade Federal de Ciências Médicas de Porto Alegre (FFFCMPA), Porto Alegre, RS.

2. Médico, cirurgião vascular. Ex-residente, Serviço de Angiologia e Cirurgia Vascular, Complexo Hospitalar Santa Casa de Porto Alegre, Porto Alegre, RS.

3. Médico residente, Serviço de Angiologia e Cirurgia Vascular, Complexo Hospitalar Santa Casa de Porto Alegre, Porto Alegre, RS.
}

Artigo submetido em 09.03.06, aceito em 06.06.06. 


\section{Introdução}

É bem conhecido que os resultados após o tratamento clínico do infarto agudo do miocárdio e após a cirurgia de revascularização cardíaca são piores nas mulheres do que nos homens ${ }^{1,2}$. No entanto, o estudo da influência do gênero sobre os resultados após cirurgia vascular periférica tem sido foco de atenção de alguns autores ${ }^{3-6}$.

A prevalência do aneurisma da aorta abdominal (AAA) émaior no homem do quena mulher 7,8 . Assim, a maioria dos estudos tem relatado os resultados principalmente nos pacientes do sexo masculino ${ }^{9}$.

Alguns trabalhos têm demonstrado que o gênero feminino influencia negativamente nos resultados após o tratamento cirúrgico do $A A A^{3,10}$; porém, existem outrostrabal hos quenão têm sustentado essa diferençal1121. Além disso, existem poucos estudos que avaliaram a influência do gênero na morbidade perioperatória e nos resultados a longo prazo após a cirurgia do AAA.

Assim, nosso objetivo foi avaliar a influência do gênero sobre a morbimortalidade cirúrgica, bem como os resultados a longo prazo de pacientes submetidos à aneurismectomia da aorta abdominal.

\section{Pacientes e métodos}

Entre dezembro de 1983 edezembro de 2003, 675 pacientes foram submetidos à cirurgia do AAA pelo primeiro autor (T .P.B.). D esses doentes, 100 (14,8\%) eram do sexo feminino. Todos os doentes eram portadores de doença aneurismática da aorta infra-renal e não-rotos.

Além da história e do exame clínico, o diagnóstico pré-operatório foi feito, fundamentalmente, através da ultra-sonografia e tomografia computadorizada de abdômen. A aortografia foi realizada em 122 pacientes (108 homens), sendo indicada na suspeita de doença oclusiva aorto-ilíaco-femoral, doença oclusiva renovascular ou visceral eenvolvimento dasartérias renais pelo aneurisma, não havendo aumento da morbidade cirúrgica dos pacientes submetidos ao exame. 0 procedimento cirúrgico foi realizado por técnica convencional através da abordagem transperitoneal da aorta abdominal pela incisão xifopúbica. 0 s pacientes permaneceram na unidade de tratamento intensivo nas primeiras 24 horas, sendo que o tempo adicional de permanência foi indicado por eventual intercorrência ou complicação.
Foram avaliadas as taxas de morbimortalidade intra-hospitalar ou em 30 dias. Além disso, aspectos cirúrgicos como tempo do procedimento, tempo do pinçamento aórtico e perda sangüínea durante a cirurgia também foram avaliados. 0 diâmetro médio dos aneurismas, o envolvimento das artérias ilíacas pela doença aneurismática e a presença do tipo inflamatório também foram estudados.

O s pacientes com alta hospitalar foram acompanhados através de um programa de seguimento clínico, feito através de consultas de revisão, questionário por via postal ou entrevista telefônica. Esta estratégia permitiu o conhecimento das taxas de sobrevida dos doentes, bem como as causas de morte durante o período pós-operatório tardio.

O s dados obtidos foram expressos em valores absolutos ou relativos (percentagens), sendo calculada a média e o desvio padrão dos valores quando necessário. A análise estatística foi feita usando-se o teste do quiquadrado $\left(\chi^{2}\right)$, teste exato de Fisher ou teste $t$ de Student quando indicado. A análise da sobrevida foi feita pela curva de Kaplan-M eier, sendo usado o teste log-rank. U m valor de $\mathrm{P}<0,05$ foi considerado significativo.

\section{Resultados}

As características clínicas e demográficas dos pacientes estão apresentadas naT abela 1 . A média de idade das mulheres foi similar à dos homens $(68,9 \pm 9,1$ versus $67,4 \pm 7,2$ anos; $P=0,089)$. Com relação às comorbidades, a hipertensão arterial foi significativamente mais comum nas mulheres ( $73 \%$ versus $62,4 \% ; P=0,042$ ), enquanto o tabagismo e a cardiopatia isquêmica foram mais freqüentes nos homens $(P<0,05)$. $N$ ão houve diferença estatisticamente significativa com relação à presença de doença cerebrovascular, insuficiência renal crônica e diabetes melito.

Com relação às características dos aneurismas, 0 diâmetro médio constatado nas mulheres foi significativamente menor do que nos homens, provavelmente relacionado com a altura menor das mulheres $(5,94 \pm 1,5$ versus $6,57 \pm 2,25 \mathrm{~cm} ; P=0,005)$. 0 envolvimento das artérias ilíacas pela doença aneurismática não diferiu entre homens e mulheres (15 versus $9 \% ; \mathrm{P}>0,05$ ), e a presença de aneurisma do tipo inflamatório foi constatado em sete mulheres $(7 \%)$ e em 30 homens $(5,2 \%)(P=0,47)$ (T abela 2$)$. 
T abela 1 - D ados demográficos e comorbidades

\begin{tabular}{lccc}
\hline Características & M ulheres (n = 100) & H omens ( $\mathbf{n}=\mathbf{5 7 5 )}$ & P \\
\hline M édia de idade (anos) & $68,9 \pm 9,1$ & $67,4 \pm 7,2$ & 0,089 \\
H ipertensão arterial & $73(73 \%)$ & $359(62,4 \%)$ & 0,042 \\
Tabagismo & $45(45 \%)$ & $412(71,6 \%)$ & $0,00 \ldots$ \\
Cardiopatia isquêmica & $14(14 \%)$ & $131(22,8 \%)$ & 0,048 \\
Diabetes melito & $6(6 \%)$ & $36(6,3 \%)$ & 0,95 \\
IRC (creatinina $\geq 2,0)$ & $4(5,1 \%)^{*}$ & $31(7,2) *$ & 0,49 \\
AIT /AVC prévio & $3(3 \%)$ & $16(2,8 \%)$ & 0,98 \\
\hline Total de 78 e 430 pacientes, respectivamente; IRC = insuficiência renal crônica (niveis de creatinina sérica maiores que \\
2 mg/dl); AIT = acidente isquêmico transitório; AVC = acidente vascular cerebral.
\end{tabular}

T abela 2 - C aracterísticas cirúrgicas

\begin{tabular}{lccc}
\hline Características & M ulheres $(\mathbf{n}=\mathbf{1 0 0})$ & H omens (n = 575) & P \\
\hline Tempo de cirurgia (min) & $205,1 \pm 57,6$ & $203,7 \pm 57,7$ & 0,676 \\
Pinçamento aórtico (min) & $45,9 \pm 16,5$ & $47,5 \pm 16,7$ & 0,349 \\
Perda sangüínea (ml) & $996,3 \pm 712,4$ & $1.026 \pm 624,8$ & 0,83 \\
M édia dos diâmetros dos AAA (cm) & $5,94 \pm 1,5$ & $6,57 \pm 2,25$ & 0,005 \\
Aneurisma de ilíacas & $9(9 \%)$ & $85(15 \%)$ & 0,12 \\
Tipo inflamatório & $7(7 \%)$ & $30(5,2 \%)$ & 0,47 \\
\hline
\end{tabular}

AAA = aneurismas da aorta abdominal.

A duração média do tempo cirúrgico foi de 205,1 $( \pm 57,6)$ minutos para as mulheres e 203,7 $( \pm 57,7)$ minutos para os homens ( $P=0,676)$. 0 tempo de pinçamento aórtico $(45,9 \pm 16,5$ versus $47,5 \pm 16,7$ minutos) e a perda sangüínea durante a cirurgia (996,3 $\pm 712,4$ versus $1.026 \pm 624,8$ ml) também não diferiram entre as mulheres e os homens, respectivamente ( $P>0,05)$ (T abela 2).

A mortalidade perioperatória global foi de 2,8\%, sendo de $4 \%$ nas mulheres e $2,6 \%$ nos homens $(P=0,43)$. A morbidade cirúrgica acometeu $14 \%$ dos pacientes do sexo feminino e $18,4 \%$ dos pacientes masculinos ( $P=0,28)$ (T abela 3$)$. As principais causas deóbitos entre os dois gêneros deveram-seà manifestação de cardiopatia isquêmica. $\mathrm{N}$ ão houve diferença nas complicações perioperatórias, sendo as intercorrências cardiopulmonares e gastrintestinais as mais comuns para ambos os grupos (T abela 3).

O s pacientes com alta hospitalar $(n=656)$ formaram uma coorte retrospectiva deste estudo. D urante 0 seguimento clínico, 38 pacientes $(5,8 \%)$ foram perdidos, sendo 32 homense 6 mulheres. A taxadesobrevida em 1, 3, 5 e 10 anos foi de $89,82,71$ e $52 \%$ para as mulheres e de $87,82,72$ e $56 \%$ para os homens, respectivamente. Pelaanálise da curvadesobrevida, não houve diferença estatística significativa entre as taxas obtidas nos diferentes grupos (Figura 1). As causas de mortetardia, duranteo seguimento clínico dos doentes, estão listadas na T abela 4. D urante o seguimento por períodos variáveis, 34 mulheres $(37,8 \%$ ) e 228 homens $(43,2 \%)$ morreram. Em 10 pacientes $(3,8 \%)$, sendo todos do sexo masculino, a causa deóbito tardio não foi 
Tabela 3 - M orbimortalidade perioperatória e suas causas

\begin{tabular}{lccc}
\hline & M ulheres $(\mathbf{n}=\mathbf{1 0 0})$ & H omens ( $\mathbf{n}=\mathbf{5 7 5})$ & $\mathbf{P}$ \\
\hline M ortalidade & $4(4 \%)$ & $15(2,6 \%)$ & 0,43 \\
Cardiopatia isquêmica & 2 & 5 & \\
Falência de múltiplos órgãos & 1 & 2 & \\
Trombose mesentérica & 1 & 2 & \\
Embolia pulmonar & - & 1 & \\
AVC & - & 2 & 0,28 \\
Sepse/infecção & - & 3 & \\
M orbidade & $14(14 \%)$ & $106(18,4 \%)$ & \\
Cardíacas & $5(5)$ & $34(5,9)$ & \\
Pulmonares & $6(6)$ & $31(5,3)$ & \\
Renais & $2(2)$ & $6(1)$ & \\
G astrintestinais & $6(6)$ & $26(4,4)$ & \\
Infecciosas & $2(2)$ & $14(2,4)$ & \\
Vasculares & $2(2)$ & $16(2,7)$ & \\
\hline
\end{tabular}

possível deser determinada. Entreascausasconhecidas, $55,9 \%$ das mulherese $47,2 \%$ doshomensmorreram de doenças cardiovasculares. $\mathrm{N}$ as mulheres, a causa mais relevante de óbito tardio foi a insuficiência renal. N os

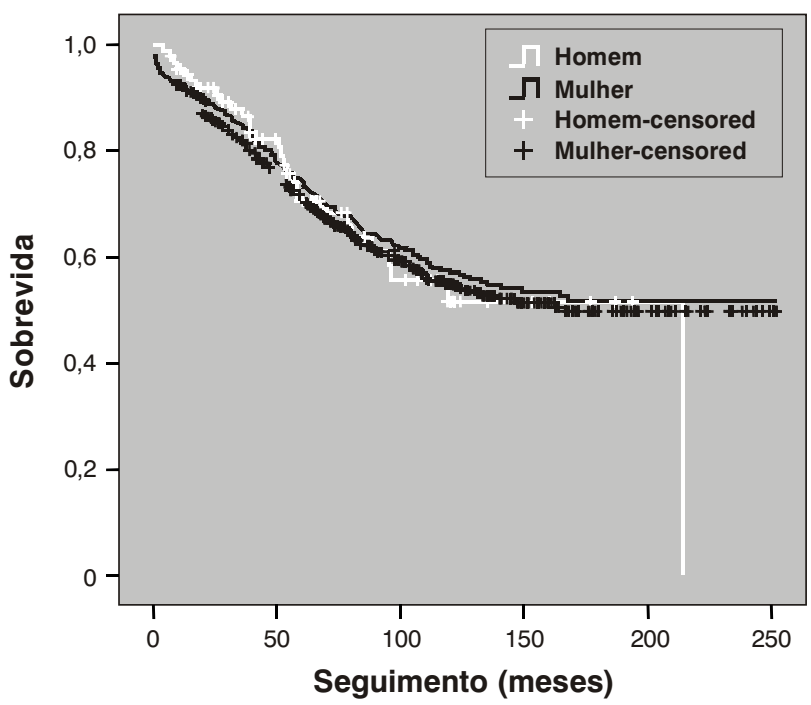

Figura 1 - Curva de sobrevida de homens e mulheres submetidos à cirurgia do aneurisma da aorta abdominal homens, houve um predomínio das neoplasias e das doenças respiratórias crônicas.

\section{Discussão}

O s principais fatores de risco para AAA são sexo masculino, idade superior a 65 anos de idadee história de tabagismo ${ }^{13}$. 0 utros fatores associados são história familiar, cardiopatia isquêmica, hipertensão arterial, doença cerebrovascular e estatura alta. As mulheres, sobretudo as diabéticas e da raça negra, têm risco reduzido para o desenvolvimento do $A A A^{13}$.

0 conhecimento dos resultados diferenciados da cirurgiado AAA entrehomensemulhereséimportante, pois dá orientação para as estratégias terapêuticas e de rastreamento. Sendo a maioria dos pacientes tratados do sexo masculino, poderia haver uma generalização dosresultados ${ }^{4}$. 0 sexo feminino respondepor até $20 \%$ dos pacientes submetidos à cirurgia do AAA. A cirurgia eletiva e de urgência do AAA pode ser de três a cinco vezes mais freqüente nos homens ${ }^{5}$.

N os estudos derastreamento, a prevalência deAAA em homens varia de 4 a $9 \%$, e em mulheres é de até $1 \%{ }^{13}$. Em nosso meio, nos cardiopatasisquêmicoscom revascularização miocárdica prévia, essa prevalência ficou próxima a $7 \%{ }^{14}$. No estudo AD AM (Aneurysm $D$ etection and $M$ anagement), a prevalência de $A A A$ em 
T abela 4 - C Causas de óbitos tardios em pacientes submetidos à cirurgia do aneurisma da aorta abdominal

\begin{tabular}{lcc}
\hline Causas & M ulheres & H omens \\
\hline Número de pacientes & 100 & 575 \\
Ó bitos no perioperatório & $4(4 \%)$ & $15(2,6 \%)$ \\
Pacientes com alta hospitalar & 96 & 560 \\
"Perdidos" durante seguimento & $6(6,25 \%)$ & $32(5,7 \%)$ \\
Pacientes com seguimento & 90 & 528 \\
M ediana de seguimento & 66 meses & 65 meses \\
Ó bitos durante seguimento & $34(\%)$ & $228(\%)$ \\
Causas conhecidas de óbitos & & \\
Cardiovasculares & $19(55,9 \%)$ & $103(47,2 \%)$ \\
Respiratórias & $2(5,9 \%)$ & $30(13,8 \%)$ \\
N eoplásicas & $2(5,9 \%)$ & $39(17,9 \%)$ \\
Renais & $4(11,8 \%)$ & $9(4,1 \%)$ \\
Infecciosas & $0(0)$ & $7(3,2 \%)$ \\
O utras & $7(20,6)$ & $30(13,8 \%)$ \\
Causas desconhecidas & $0(0)$ & $10(4,4 \%)$ \\
\hline
\end{tabular}

mulheres foi de $1,3 \%{ }^{9}$. Com essa freqüência tão baixa de AAA nas mulheres, não se justificam programas de rastreamento nessa população, pois o número de mortesrelacionadasao AAA quepodem ser prevenidasnesse grupo de doentes é baixo ${ }^{13}$. Já a Society of Vascular Surgery e a Society for $V$ ascular M edicine and Biology recomendam 0 rastreamento do AAA em homens $e$ mulheres com idade entre 60 e 85 anos com fatores de risco cardiovasculares e para os homens e mulheres acima de 50 anos com história familiar de $A A A^{15}$. N 0 estudo AD AM , a história familiar de AAA e a presença de doença cerebrovascular foram mais freqüentes nas mulheres ${ }^{9}$.

A influência do gênero sobre os resultados após a cirurgia vascular periférica já foi estudada em alguns ensaios prévios. Com relação à doença carotídea, constatou-sequea sua prevalênciaémaisbaixa nas mulheres do que nos homens, com uma proporção de 3:2 até $8: 1^{6}$, e a proporção dos procedimentos na artéria carótida em pacientes do sexo feminino variando de 27 a $42 \% 16,17$. Com relação aos resultados pós-operatórios, a maioria dos estudos indica um pior resultado nas mulheres, com uma taxa de acidente vascular cerebral maior no sexo feminino 18,19 .

$\mathrm{N}$ o que diz respeito às cirurgias de revascularização infra-inguinal, cerca de $1 / 3$ dos procedimentos são realizados em mulheres, com taxas de mortalidade perioperatória e perviedade do enxerto a médio elongo prazo significativamente inferiores aos pacientes do gênero masculino 20,21 .

A causa para os piores resultados obtidos no gênero feminino após a cirurgia do AAA é desconhecida. Presume-se que possa existir uma maior comorbidade nesse grupo de pacientes. $N$ esse contexto, um estudo envolvendo 582 pacientes com 92 mulheres não demonstrou diferença nos fatores de risco 22 ; no entanto, um estudo populacional, com 5.419 pacientes, demonstrou queas mulheres possuíam uma comorbidade cardiovascular maior que oshomen $s^{6}$. 0 utra causa seria o uso de diâmetros semel hantes do AAA para a conduta e indicação cirúrgica nos diferentes gêneros. Isso possibilitaria uma indicação cirúrgica maistardia nas mulheres, pois elas são portadoras de aortas com menor diâmetro. 0 ideal seria considerar o tratamento cirúrgico do AAA de maneira distinta para os dois grupos, como concluiu o UK Small Aneurysm Trial, em que a cirurgia estaria indicada para AAA com dimensões acima de 4,5 cm nas mulheres e 5,5 cm noshomens $\mathrm{s}^{23}$.

Com relação aos resultados de nosso estudo, constatamos que as mulheres tiveram uma média de idade um pouco maior que os homens e demonstraram uma freqüência maior de hipertensão arterial e aneurismas 
menores. Esses achados são similares àqueles obtidos por outrosautores $3,5,24-26$. N o entanto, há autores que não demonstraram uma diferença estatística significativa nas comorbidades entre os gêneros $s^{9,22}$.

Existem poucos estudos que relatam as diferenças na morbidade perioperatória entre os gêneros após a correção do AAA. N ossos resultadosnão demonstraram diferença estatisticamente significativa na morbidade cirúrgica entre os gêneros, sendo similares aos dados obtidos pelo estudo de Johnston ${ }^{3}$. Com relação à mortalidadeperioperatória, o óbito ocorreu em $4 \%$ das mulheres e 2,6\% nos homens, não havendo, também, diferença significativa, concordando com os resultados de outros autores $3,10,22$; porém, outros estudos têm demonstrado um maior risco de óbito nas mulheres. Assim, Katz et al., com basenos dados demais de 8.000 pacientes operados no estado de M ichigan entre 1980 e 1990, demonstraram uma mortalidade maior no grupo feminino ${ }^{11}$. Da mesma forma, analisando os resultadosatravés do $\mathrm{N}$ ati onwidel npatient Samplenorteamericano, D imick et al. obtiveram uma mortalidade significativamentemaior nasmulheres (5,3 versus $3,2 \%$ ). Além disso, esses autorestambém demonstraram queos piores resultados foram obtidos em pacientes do sexo feminino, com idade superior a 65 anos e operados em hospitais de baixo volume $(7,1 \%)$, sendo os melhores resultados obtidosem pacientesmasculinos, com idade inferior a 65 anos e operados em hospitais de grande volume $(0,8 \%)^{12}$.

$\mathrm{N}$ orman et al. ${ }^{27}$ revisaram 32 trabalhos sobre os resultados após a cirurgia do AAA, englobando mais de 10.500 pacientes, com uma mortal idade variando de 0 a 10,4\% e sobrevida a longo prazo, em média, de 70\% em 5 anos, comparada com $80 \%$ da população em geral. Apenas três estudos relataram a comparação da sobrevida a longo prazo entre homens e mulheres. Em nenhum desses estudos constatou-se uma diferença significativa na sobrevida em 5 anos entre os gêneros, variando de 61 a $79 \%$ nas mulheres e de 63 a $79 \%$ nos homens ${ }^{10,28,29}$. 0 estudo canadense de J ohnston também não demonstrou diferença significativa na sobrevida em 5 anos entre homens $(82,8 \%)$ e mulheres $(74,2 \%)^{3}$. N ossos resultados estão de acordo com esses achados, pois a sobrevida em nossos pacientes do sexo feminino (71\%) são semelhantes àquela obtida nos pacientes masculinos (72\%) (Tabela 5). N o entanto, quando analisada a sobrevida relativa em 5 anos (considerada a maneira mais fidedigna dos resultados a longo prazo, poisé uma relação com a população geral de mesma idade, sexo e localidade), $\mathrm{N}$ orman et al. obtiveram piores resultados a longo prazo no sexo feminino, quando comparado com o grupo masculino ${ }^{10}$. A chados semel hantes foram aqueles obtidos por Stenbeak et al. ${ }^{4}$, em que a sobrevida em 5 anos foi similar entreosgêneros; no entanto, a sobrevida relativa foi pior nas mulheres. Em nossos pacientes, a principal causa de óbito tardio, em ambos os gêneros, foi devido à doença cardiovascular, seguida das doenças neoplásicas, principalmente nos homens, concordando com a revisão de N orman et al. ${ }^{27}$.

Embora não seja assunto de nosso trabalho, a influência do gênero nos resultados após a cirurgia do AAA roto também foi estudada por outros autores. Assim, Evans et al. ${ }^{30}$, através dos dados de um sistema de auditoria de resultados da Inglaterra, analisaram quase 700 pacientes submetidos à cirur-

Tabela 5 - Resultados a longo prazo do tratamento eletivo do aneurisma da aorta abdominal em homens e mulheres

\begin{tabular}{|c|c|c|c|c|c|c|}
\hline \multirow[t]{2}{*}{ Ano } & \multirow[t]{2}{*}{ Estudo } & \multirow[t]{2}{*}{ Período } & \multirow[t]{2}{*}{ Casos } & \multirow{2}{*}{$\begin{array}{c}\text { Ó bito } \\
\text { perioperatório }\end{array}$} & \multicolumn{2}{|c|}{ Sobrevida 5 anos } \\
\hline & & & & & $M$ & $\mathbf{F}$ \\
\hline 1981 & Crawford ${ }^{28}$ & $1955-80$ & $737 \mathrm{M}+123 \mathrm{~F}$ & $4,8 \%$ & $63 \%$ & $61 \%$ \\
\hline 1994 & Johnston ${ }^{3}$ & - & $545 M+134 F$ & $4,4 \% M ; 5,2 \% F$ & $82,8 \%$ & $74,2 \%$ \\
\hline 1995 & Soisalon-Soininen 29 & $1970-92$ & $595 \mathrm{M}+111 \mathrm{~F}$ & $7,4 \%$ & $70 \%$ & $72 \%$ \\
\hline 1998 & N orman 10 & 1985-94 & $796 M+139 F$ & $4,4 \% \mathrm{M} ; 3,6 \% \mathrm{~F}$ & $79 \%$ & $79 \%$ \\
\hline 2005 & Bonamigo & $1983-2003$ & $575 \mathrm{M}+100 \mathrm{~F}$ & $2,8 \% \mathrm{M} ; 4 \% \mathrm{~F}$ & $71 \%$ & $72 \%$ \\
\hline
\end{tabular}

$F=$ feminino; $M=$ masculino. 
gia do AAA roto entre1983 e1995, não demonstrando diferença na mortalidade perioperatória e na sobrevida a longo prazo entre os dois gêneros. N o estudo de Johnston, a mortalidade foi de $55 \%$ nos homense 49,2\% nas mulheres ${ }^{3}$. Ausência de diferença na mortalidade após a correção do AAA roto também foi obtida por outrosautores 31,32 . Por outro lado, há autores que demonstraram uma diferença entre os gêneros nos resultados após a cirurgia do AAA roto. D esta maneira, Semmens et al. 33 identificaram uma mortalidade de $76 \%$ para os homens e $90 \%$ para as mulheres $(P<0,0001)$. Resultados semel hantes também foram obtidos por J ohansen et al. ${ }^{34}$ (67 versus $90 \%$ ). Em estudo recente, feito por D ueck et al. ${ }^{35}$, demonstrou-se que o gênero feminino não foi considerado um fator independente para um resultado pior em pacientes operados eletivamente; no entanto, em pacientes operados de AAA roto, o sexo feminino mostrou-se um fator independente para um pior desfecho perioperatório.

Com relação ao tratamento endovascular, 0 uriel et al. ${ }^{36}$ demonstraram resultados semel hantes entre homens e mulheres após o tratamento do AAA, com uma mortalidade perioperatória de 1,3 e 3,1\%, respectivamente. Esses achados concordam com aqueles obtidos por $M$ athison et al. ${ }^{26}$, queobtiveram uma mortalidade de $2,8 \%$ para os homens e $0,8 \%$ para as mulheres $(P>0,05)$, porém com uma taxa maior de sucesso no procedimento nos homens e uma percentagem maior de procedimentos abandonados no grupo feminino. No estudo de Sampaio et al. ${ }^{24}$, não houve diferença na mortalidade perioperatória, na sobrevida e na incidência de endoleak a médio prazo.

Em resumo, observamos que a prevalência da cirurgia eletiva do AAA foi quase seis vezes mais comum nos homens. $\mathrm{N}$ o entanto, as taxas de morbidade e mortal idade perioperatórias foram semelhantes entre os gêneros, apesar de as mulheres apresentarem hipertensão arterial com maior freqüência e possuírem aneurismas menores do que os homens, e estes serem, com maior freqüência, cardiopatas isquêmicos e tabagistas. Além disso, os resultados da sobrevida a longo prazo não diferiram entre os gêneros, com cerca da metade dos óbitos tardios ocorrendo por doença cardiovascular em ambos os grupos. Assim, o conhecimento dos resultados cirúrgicosnos pacientes de ambos os gêneros possibilita uma meIhor racionalização da conduta frente ao paciente com AAA.

\section{R eferências}

1. M arrugat J, Sala J, M asia R, et al. M ortality differences between men and women following first myocardial infarction. JAM A. 1998;280:1405-9.

2. Khan SS, N essim S, Gray R, C zer LS, Chaux A, M atloff J. Increased mortality of women in coronary artery bypass surgery: evidencefor referral bias. Ann Intern M ed. 1990;112: 561-7.

3. Johnston $\mathrm{KW}$. Influence of sex on the results of abdominal aortic aneurysm repair. C anadian Society for V ascular Surgery Aneurysm Study G roup. J V asc Surg. 1994;20:914-23.

4. Stenbaek J, Granath F, Swedenborg J. O utcome after abdominal aortic aneurysm repair: difference between men and women. Eur J V asc Endovasc Surg. 2004;28:47-51.

5. Katz DJ, Stanley JC, Zelenock GB. Gender differences in abdominal aortic aneurysm preval ence, treatment and outcome. J V asc Surg. 1997;25:561-8.

6. Norman PE, Semmens JB, Lawrence-Brown M, H olman $C D$. Theinfluence of gender on outcomefollowing peripheral vascular surgery: a review. Cardiovasc Surg. 2000;8:111-5.

7. Blanchard JF. E pidemiology of abdominal aortic aneurysms. Epidemiol Rev. 1999;21:207-21.

8. Lederle FA, Johnson GR, W ilson SE, et al. The aneurysm detection and management study screening program: validation cohort and final results. Arch Intern M ed. 2000;160:1425-30.

9. Lederle FA, Johnson GR, W ilson SE, Aneurysm D etection and $M$ anagement $V$ eterans Affairs Cooperative Study. Abdominal aortic aneurysm in women. J V asc Surg. 2001;34: 122-6.

10. N orman PE, Semmens JB, Lawrence-Brown M M, H olman $C D$. Long term relative survival after surgery for abdominal aortic aneurysm in western Australia: population based study. BM J. 1998;317:852-6.

11. Katz DJ, Stanley JC, Zelenock G B. O perative mortality rates for intact and ruptured abdominal aortic aneurysms in M ichigan: an eleven-year statewide experience. J V asc Surg. 1994;19:804-15.

12. D imick JB, Stanley JC, Axelrod D A, et al. Variation in death rate after abdominal aortic aneurysmectomy in the $U$ nited States: impact of hospital volume, gender and age. Ann Surg. 2002;235:579-85.

13. U .S. PreventiveServices T ask Force. Screening for abdominal aortic aneurysm: recommendation statement. Ann Intern M ed. 2005;142:198-202.

14. Bonamigo TP, Siqueira I. Screening for abdominal aortic aneurysms. R evH osp Clin FacM ed São Paulo. 2003;58:63-8.

15. Kent $K, Z$ wolak RM , Jaff M R, et al. Screening for abdominal aortic aneurysm: a consensusstatement. J V asc Surg. 2004;39: 267-9.

16. M RC European Carotid Surgery Trial: interim results for symptomatic patients with severe (70-99\%) or with mild (029\%) carotid stenosis. European Carotid Surgery Trialists' Collaborative Group. Lancet. 1991;337:1235-43.

17. M axwell J, RutledgeR, C ovington D L, Churchill M P, Clancy TV. A statewide, hospital-based analysis of frequency and outcomes in carotid endarterectomy. Am J Surg. 1997;174: 655-60; discussion 660-1. 
18. Schneider JR, D rosteJ S, G olan JF. C arotid endarterectomy in women versus men: patient characteristics and outcomes. J V asc Surg. 1997;25:890-6.

19. H ertzer NR, O 'H ara PJ, M ascha EJ, Krajewski LP, Sullivan TM , Beven EG . Early outcomeassessment for 2228 consecutive carotid endarterectomy procedures: the Cleveland Clinic experience from 1989 to 1995. J V asc Surg. 1997;26:1-10.

20. Enzler M A, Ruoss $M$, Seifert $B$, Berger $M$. The influence of gender on the outcome of arterial procedures in the lower extremity. Eur J Vasc Endovasc Surg. 1996;11:446-52.

21. M agnant J G, C ronenwett JL, W alsh D B, Schneider JR, Besso $S R$, Z wolak RM. Surgical treatment of infrainguinal arterial occlusive disease in women. J Vasc Surg. 1993;17:67-76; discussion 76-8.

22. Starr JE, H ertzer N R, M aschaEJ, et al. I nfluence of gender on cardiac risk and survival in patients with infrarenal aortic aneurysms. J Vasc Surg. 1996;23:870-80.

23. Brown LC, Powell JT. Risk factors for rupture in abdominal aortic aneurysms under surveillance. UK Small Aneurysm Trial Participants. Ann Surg. 1999;230:289-96; discussion 296-7.

24. Sampaio SM , Panneton JM , M ozes GI, et al. Endovascular abdominal aortic aneurysm repair: does gender matter? Ann V asc Surg. 2004;18:653-60.

25. Parlani $G$, Verzini $F, Z$ annetti $S$, et al. D oes gender influence outcome of AAA endoluminal repair? Eur J Vasc Endovasc Surg. 2003;26:69-73.

26. $M$ athison $M$, Becker $G J, K$ atzen $B T$, et al. The influence of female gender on the outcome of endovascular abdominal aortic aneurysm repair. J V asc I nterv Radiol. 2001;12:1047-51.

27. N orman PE, Semmens] B, Lawrence-Brown M M . Long-term relative survival following surgery for abdominal aortic aneurysm: a review. Cardiovasc Surg. 2001;9:219-24.

28. Crawford ES, Saleh SA, Babb JW 3rd, Glaeser D H, V accaro PS, Silvers A. Infrarenal abdominal aortic aneurysm: factors influencing survival after operation performed over a 25-year period. Ann Surg. 1981;193:699-709.
29. Soisalon-Soininen $S$, Salo JA, Takkunen $O, M$ attila $S$. Comparison of long term survival after repair of ruptured and non-ruptured abdominal aortic aneurysm. V asa. 1995;24:42-8.

30. Evans SM , Adam D J, Bradbury AW. The influence of gender on outcomeafter ruptured abdominal aortic aneurysm. J V asc Surg. 2000;32:258-62.

31. G loviczki P, Pairolero PC, M ucha P Jr. Ruptured abdominal aortic aneurysms: repair should not be denied. J V asc Surg. 1992;15:851-7; discussion 857-9.

32. Bauer EP, Redaelli C, von Segesser LK, T urina M I. Ruptured abdominal aorticaneurysms: predictorsfor early complications and death. Surgery. 1993;114:31-5.

33. Semmens JB, N orman PE, Lawrence-Brown M M, H olman $C D$. Influence of gender on outcomefrom ruptured abdominal aortic aneurysm. Br J Surg. 2000;87:191-4.

34. Johansen K, Kohler TR, Nicholls SC, Zierler RE, Clowes AW, Kazmers A. Ruptured abdominal aortic aneurysm: the H arborview experience. J V asc Surg. 1991;13:240-5; discussion 245-7.

35. D ueck AD, Kucey DS, Johnston KW, Alter D, Laupacis A. Survival after ruptured abdominal aortic aneurysm: effect of patient, surgeon, and hospital factors. J Vasc Surg. 2004;39:1253-60.

36. O uriel K, Greenberg RK, Clair DG, et al. Endovascular aneurysm repair: gender specific results. J V asc. 2003;38:93-8.

Correspondência:

T elmo Pedro Bonamigo

Rua C oronel Bordini, 675/303

CEP 90440-002 - Porto Alegre, RS

Tel./Fax.: (51) 3333.1642

E-mail: telmobonamigo@terra.com.br 